\title{
Dynamic Analysis of a Delayed Reaction-Diffusion Predator-Prey System with Modified Holling-Tanner Functional Response
}

\author{
Xinhong Pan, ${ }^{1,2}$ Min Zhao,, ${ }^{2,3}$ Chuanjun Dai, ${ }^{2,3}$ and Yapei Wang ${ }^{1,2}$ \\ ${ }^{1}$ School of Mathematics and Information Science, Wenzhou University, Wenzhou, Zhejiang 325035, China \\ ${ }^{2}$ Zhejiang Provincial Key Laboratory for Water Environment and Marine Biological Resource Protection, \\ Wenzhou University, Wenzhou, Zhejiang 325035, China \\ ${ }^{3}$ School of Life and Environmental Science, Wenzhou University, Wenzhou, Zhejiang 325035, China
}

Correspondence should be addressed to Min Zhao; zmcn@tom.com

Received 21 May 2014; Accepted 21 July 2014

Academic Editor: Sanling Yuan

Copyright (C) 2015 Xinhong Pan et al. This is an open access article distributed under the Creative Commons Attribution License, which permits unrestricted use, distribution, and reproduction in any medium, provided the original work is properly cited.

\begin{abstract}
A predator-prey model with modified Holling-Tanner functional response and time delays is considered. By regarding the delays as bifurcation parameters, the local and global asymptotic stability of the positive equilibrium are investigated. The system has been found to undergo a Hopf bifurcation at the positive equilibrium when the delays cross through a sequence of critical values. In addition, the direction of the Hopf bifurcation and the stability of bifurcated periodic solutions are also studied, and an explicit algorithm is obtained by applying normal form theory and the center manifold theorem. The main results are illustrated by numerical simulations.
\end{abstract}

\section{Introduction}

The dynamic relationship between prey and predators has long been and will continue to be one of the dominant subjects in mathematical ecology due to its universal existence and importance [1-12]. In [13, 14], the author proposed the following predator-prey model based on the model in May [15]:

$$
\begin{gathered}
\frac{d u}{d t}=r u\left(1-\frac{u}{K}\right)-p(u) v, \\
\frac{d v}{d t}=v\left[s\left(1-\frac{h v}{u}\right)\right],
\end{gathered}
$$

where $u$ and $v$ denote the population of prey and predator, respectively, and $r$ and $s$ are the intrinsic growth rates of prey and predator, respectively. The parameter $K$ represents the carrying capacity of the prey and the ratio $u / h$ represents the carrying capacity of the predator. It has been assumed that both prey and predator populations grow logistically and that the predator consumes the prey according to a functional $p(u)$.
In recent years, models with time delay have been extensively studied by many authors $[5,16-26]$. The authors of [1] discussed model (1) with a discrete delay:

$$
\begin{gathered}
\frac{d u}{d t}=r u\left(1-\frac{u(t-\tau)}{K}\right)-m u v, \\
\frac{d v}{d t}=v\left[s\left(1-\frac{h v}{u}\right)\right]
\end{gathered}
$$

and obtained the stability of equilibria, the existence of Hopf bifurcation, and the direction of bifurcating periodic solutions. This paper focuses mainly on the effects of both spatial diffusion and time delay on system (1). It is assumed that the delay affects predation and consumption and that the system has homogeneous Neumann boundary conditions:

$$
\begin{gathered}
\frac{\partial u}{\partial t}=d_{1} \Delta u+r u\left(1-\frac{u}{K}\right)-\frac{m u^{2} v\left(t-\tau_{1}\right)}{a+u^{2}}, \quad x \in \Omega, t>0, \\
\frac{\partial v}{\partial t}=d_{2} \Delta v+v\left[s\left(1-\frac{h v}{u\left(t-\tau_{2}\right)}\right)\right], \quad x \in \Omega, t>0,
\end{gathered}
$$




$$
\begin{array}{cc}
\frac{\partial u}{\partial n}=\frac{\partial v}{\partial n}=0, & x=\partial \Omega, t>0, \\
u(x, t)=u_{0}(x, t), & x \in \Omega, t \in\left[-\tau_{2}, 0\right], \\
v(x, t)=v_{0}(x, t), & x \in \Omega, t \in\left[-\tau_{1}, 0\right],
\end{array}
$$

where $d_{1}$ and $d_{2}$ are the diffusion coefficients of prey and predator, respectively, $\Delta=\partial^{2} / \partial x^{2}$ denotes the Laplacian operator, and $n$ is the outward unit normal vector on $\partial \Omega$. For convenience, it is assumed that $\Omega=(0, l \pi), l>0$ and that all parameters are positive.

The rest of this paper is structured as follows. In Section 2, the local stability of equilibria is analyzed using the associated characteristic equations, and the occurrence of the Hopf bifurcation with time delays is presented. In Section 3, the global asymptotical stability of the interior equilibrium for any $\tau_{1}, \tau_{2} \geq 0$ is proved by means of the upper-lower solution method. In Section 4, using normal form theory and the center manifold theorem, the stability and direction of bifurcating periodic orbits are investigated. Finally, numerical simulations and a brief discussion are presented.

\section{Local Stability and Hopf Bifurcation Analysis}

In this section, the local stability of the equilibria of system (3) is analyzed. Denote

$$
\begin{gathered}
X=C\left([0, l \pi], R^{2}\right), \\
\langle u, v\rangle=\left\langle u_{1}, v_{1}\right\rangle+\left\langle u_{2}, v_{2}\right\rangle, \quad \tau=\tau_{1}+\tau_{2},
\end{gathered}
$$

for $u=\left(u_{1}, u_{2}\right), v=\left(v_{1}, v_{2}\right) \in X$; then $(X,\langle\cdot, \cdot\rangle)$ is a Hilbert space. In the abstract space $C([-\tau, 0], X)$, system (3) can be regarded as an abstract functional differential equation.

System (3) has two nonnegative equilibria $(K, 0)$ and $\left(u^{*}, v^{*}\right)$, where

$$
v^{*}=\frac{1}{h} u^{*}
$$

and $u^{*}$ is the positive root of the equation

$$
r h u^{3}+(m K-r h K) u^{2}+a r h u-a r h K=0
$$

Let $A(u)=r h u^{3}+(m K-r h K) u^{2}+a r h u-a r h K$; then $A(0)=$ $-\operatorname{arh} K<0, A(K)=m K^{3}>0$, which guarantee the existence of $u^{*} \in(0, K)$.

From analysis of the characteristic equation of $(K, 0)$, it can easily be determined that it always has a saddle point. To analyze the stability of the positive equilibrium $\left(u^{*}, v^{*}\right)$, the first step is to linearize system $(3)$ at $\left(u^{*}, v^{*}\right)$ :

$$
\frac{\partial U(t)}{\partial t}=d \Delta U(t)+L\left(U_{t}\right)
$$

where $d \Delta=\left(d_{1} \Delta, d_{2} \Delta\right)$,

$$
\begin{gathered}
\operatorname{dom}(d \Delta)=\left\{(u, v)^{T}: u, v \in C^{2}([0, l \pi], R),\right. \\
\left.\frac{\partial u}{\partial x}=\frac{\partial v}{\partial x}=0, x=0, l \pi\right\},
\end{gathered}
$$

and $L: C([-\tau, 0], X) \rightarrow X$ is defined as

$L(\phi)$

$$
=\left(\begin{array}{c}
{\left[r-\frac{2 r u^{*}}{K}-\frac{2 a m u^{*} v^{*}}{\left(a+u^{* 2}\right)^{2}}\right] \phi_{1}(0)-\frac{m u^{*}}{a+u^{* 2}} \phi_{2}\left(-\tau_{1}\right)} \\
\frac{s}{h} \phi_{1}\left(-\tau_{2}\right)-s \phi_{2}(0)
\end{array}\right)
$$

for $\phi=\left(\phi_{1}, \phi_{2}\right)^{T} \in C([-\tau, 0], X)$.

The characteristic equation of (7) is

$$
\lambda y-d \Delta y-L\left(e^{\lambda} y\right)=0, \quad y \in \operatorname{dom}(d \Delta), \quad y \neq 0 .
$$

Recall that $-\Delta$ under the Neumann boundary condition has eigenvalues $0=\mu_{0}<\mu_{1} \leq \mu_{2} \leq \cdots \leq \mu_{n} \leq \mu_{n+1} \leq \cdots$ and $\lim _{i \rightarrow \infty} \mu_{i}=\infty$, with the corresponding eigenfunctions $\psi_{n}(x)$. Substituting

$$
y=\sum_{n=0}^{\infty} \psi_{n}(x)\left(\begin{array}{l}
y_{1 n} \\
y_{2 n}
\end{array}\right)
$$

into (10), the following expression results:

$$
\begin{aligned}
& \left(\begin{array}{cc}
r-\frac{2 r u^{*}}{K}-\frac{2 a m u^{*} v^{*}}{\left(a+u^{* 2}\right)^{2}}-\mu_{n} d_{1} & -\frac{m u^{*}}{a+u^{* 2}} e^{-\lambda \tau_{1}} \\
\frac{s}{h} e^{-\lambda \tau_{2}} & -s-\mu_{n} d_{2}
\end{array}\right)\left(\begin{array}{l}
y_{1 n} \\
y_{2 n}
\end{array}\right) \\
& =\lambda\left(\begin{array}{l}
y_{1 n} \\
y_{2 n}
\end{array}\right) .
\end{aligned}
$$

Hence, it can be concluded that the characteristic equation (10) is equivalent to the equation

$$
\lambda^{2}+A_{n} \lambda+B_{n}+C e^{-\lambda \tau}=0, \quad n=0,1,2, \ldots,
$$

where

$$
\begin{gathered}
A_{n}=\mu_{n}\left(d_{1}+d_{2}\right)-r+\frac{2 r u^{*}}{K}+\frac{2 a m u^{*} v^{*}}{\left(a+u^{* 2}\right)^{2}}+s, \\
B_{n}=\left(r-\frac{2 r u^{*}}{K}-\frac{2 a m u^{*} v^{*}}{\left(a+u^{* 2}\right)^{2}}-\mu_{n} d_{1}\right)\left(-s-\mu_{n} d_{2}\right), \\
C=\frac{s}{h} \frac{m u^{*}}{a+u^{* 2}}, \quad \tau=\tau_{1}+\tau_{2} .
\end{gathered}
$$

The stability of the positive equilibrium $\left(u^{*}, v^{*}\right)$ can be determined by the distribution of the roots of (13). It is locally 
asymptotically stable if all the roots of (13) have negative real parts for all $n=0,1,2, \ldots$ Obviously, 0 is not a root of (13) for all $n=0,1,2, \ldots$. When $\tau=0$ as well as $\tau_{1}=\tau_{2}=0$, (13) can be simplified as

$$
\lambda^{2}+A_{n} \lambda+B_{n}=0
$$

It can be verified that

$$
A_{n}>0, \quad B_{n}>0, \quad \text { if } r-\frac{2 r u^{*}}{K}<0 ;
$$

that is,

$$
u^{*}>\frac{K}{2}
$$

which requires that

$$
A\left(\frac{K}{2}\right)=\frac{m}{4} K^{3}-\frac{r h}{8} K^{3}-\frac{1}{2} \operatorname{arh} K<0
$$

or

$$
2 m-r h<0 .
$$

Therefore, the following theorem can be stated.

Theorem 1. If $2 m-r h<0$ holds, the interior equilibrium $\left(u^{*}, v^{*}\right)$ of system (3) with $\tau_{1}=\tau_{2}=0$ is asymptotically stable.

When $\tau \neq 0$, assume that $\lambda=i w_{0}\left(w_{0}>0\right)$ is a root of (13). Substituting it into (13) yields:

$$
\begin{gathered}
w_{0}^{2}-B_{n}=C \cos w_{0} \tau \\
w_{0} A_{n}=C \sin w_{0} \tau
\end{gathered}
$$

that is,

$$
w_{0}^{4}+\left(A_{n}^{2}-2 B_{n}\right) w_{0}^{2}+B_{n}^{2}-C^{2}=0
$$

where

$$
\begin{aligned}
A_{n}^{2}-2 B_{n}= & \left(-r+\frac{2 r u^{*}}{K}+\frac{2 a m u^{*} v^{*}}{\left(a+u^{* 2}\right)^{2}}+\mu_{n} d_{1}\right)^{2} \\
& +\left(s+\mu_{n} d_{2}\right)^{2}, \\
B_{n}^{2}-C^{2}= & \left(-r+\frac{2 r u^{*}}{K}+\frac{2 a m u^{*} v^{*}}{\left(a+u^{* 2}\right)^{2}}+\mu_{n} d_{1}\right)^{2} \\
& \times\left(s+\mu_{n} d_{2}\right)^{2}-\left(\frac{s}{h} \frac{m u^{*}}{a+u^{* 2}}\right)^{2} .
\end{aligned}
$$

This leads to the following theorem.

Theorem 2. If $2 m-r h<0$ and $B_{n}^{2}-C^{2}>0$ hold for $n=$ $0,1,2, \ldots$, then the interior equilibrium $\left(u^{*}, v^{*}\right)$ of system (3) is asymptotically stable for all $\tau_{1}, \tau_{2} \geq 0$.
Proof. If $B_{n}^{2}-C^{2}<0$, then there exists $N_{0} \geq 0$ such that, for $0 \leq n \leq N_{0}$, (20) has a unique positive real root:

$$
w_{0}=\sqrt{\frac{2 B_{n}-A_{n}^{2}+\sqrt{\left(A_{n}^{2}-2 B_{n}\right)^{2}-4\left(B_{n}^{2}-C^{2}\right)}}{2}} .
$$

From (20), it follows that

$$
\sin w_{0} \tau=\frac{w_{0} A_{n}}{C}, \quad \cos w_{0} \tau=\frac{w_{0}^{2}-B_{n}}{C} .
$$

Then (13) has a pair of pure imaginary roots $\pm i w_{0}$ when

$$
\tau=\tau_{k}=\tau_{0}+\frac{2 k \pi}{w_{0}}, \quad k=0,1,2 \ldots
$$

where

$$
\tau_{0}=\frac{1}{w_{0}}\left(\arccos \frac{w_{0}^{2}-B_{n}}{C}\right) .
$$

Substituting $\lambda(\tau)$ into (13) and taking the derivatives with respect to $\tau$ lead to

$$
2 \lambda \frac{d \lambda(\tau)}{d \tau}+A_{n} \frac{d \lambda(\tau)}{d \tau}+C e^{-\lambda \tau}\left(-\lambda-\tau \frac{d \lambda(\tau)}{d \tau}\right)=0
$$

For $\tau=\tau_{0}$, considering $C e^{-i w_{0} \tau}=w_{0}^{2}-B_{n}-i w_{0} A_{n}$, it follows that

$$
\begin{gathered}
\left.\operatorname{sign}\left\{\frac{d \operatorname{Re}(\lambda(\tau))}{d \tau}\right\}\right|_{\lambda=i w_{0}}=\left.\operatorname{sign}\left\{\operatorname{Re}\left(\frac{d \lambda(\tau)}{d \tau}\right)\right\}\right|_{\lambda=i w_{0}} \\
=\operatorname{sign}\left\{\operatorname{Re}\left(\frac{i C w_{0} e^{-i w_{0} \tau_{0}}}{2 w_{0} i+A_{n}-C \tau_{0} e^{-i w_{0} \tau_{0}}}\right)\right\} \\
=\operatorname{sign}\left\{\operatorname{Re}\left(\frac{i w_{0}^{3}-B_{n} w_{0} i+w_{0}^{2} A_{n}}{2 w_{0} i+A_{n}-\tau_{0}\left(w_{0}^{2}-B_{n}-i w_{0} A_{n}\right)}\right)\right\} \\
=\operatorname{sign}\left\{\operatorname{Re}\left(\frac{w_{0}^{2} A_{n}+i\left(w_{0}^{3}-B_{n} w_{0}\right)}{-\tau_{0}\left(w_{0}^{2}-B_{n}\right)+A_{n}+i\left(\tau_{0} w_{0} A_{n}+2 w_{0}\right)}\right)\right\} \\
=\operatorname{sign}\left\{\left(w_{0}^{2} A_{n}\left[-\tau_{0}\left(w_{0}^{2}-B_{n}\right)+A_{n}\right]\right.\right. \\
\left.+\left(w_{0}^{3}-B_{n} w_{0}\right)\left(\tau_{0} w_{0} A_{n}+2 w_{0}\right)\right) \\
\times\left(\left[-\tau_{0}\left(w_{0}^{2}-B_{n}\right)+A_{n}\right]^{2}\right. \\
\left.\left.+\left[\tau_{0} w_{0} A_{n}+2 w_{0}\right]^{2}\right)^{-1}\right\}
\end{gathered}
$$




$$
\begin{gathered}
=\operatorname{sign}\left\{\frac{w_{0}^{2}\left[A_{n}^{2}+2 w_{0}^{2}-2 B_{n}\right]}{\left[-\tau_{0}\left(w_{0}^{2}-B_{n}\right)+A_{n}\right]^{2}+\left[\tau_{0} w_{0} A_{n}+2 w_{0}\right]^{2}}\right\} \\
=\operatorname{sign}\left\{w_{0}^{2} \cdot \frac{2 B_{n}-A_{n}^{2}+\sqrt{\left(A_{n}^{2}-2 B_{n}\right)^{2}-4\left(B_{n}^{2}-C^{2}\right)}}{2}\right. \\
\times\left(\left[-\tau_{0}\left(w_{0}^{2}-B_{n}\right)+A_{n}\right]^{2}\right. \\
\left.\left.+\left[\tau_{0} w_{0} A_{n}+2 w_{0}\right]^{2}\right)^{-1}\right\}=1 .
\end{gathered}
$$

In other words, $\left.(d \lambda / d \tau)\right|_{\tau=\tau_{0}}>0$. stated.

From the above discussion, the following theorem can be

Theorem 3. If $B_{n}^{2}-C^{2}<0$ holds, then the following statements are true.

(1) If $0 \leq \tau_{1}+\tau_{2}<\tau_{0}$, then the interior equilibrium $\left(u^{*}, v^{*}\right)$ of system (3) is asymptotically stable.

(2) If $\tau_{1}+\tau_{2}>\tau_{0}$, then the interior equilibrium $\left(u^{*}, v^{*}\right)$ of system (3) is unstable.

(3) System (3) undergoes a Hopf bifurcation at the interior equilibrium $\left(u^{*}, v^{*}\right)$ for $\tau_{1}+\tau_{2}=\tau_{k}, k=0,1,2, \ldots$

\section{Global Stability}

This section mainly proves that the interior equilibrium $\left(u^{*}, v^{*}\right)$ is globally asymptotically stable with the upperlower solution method in [27, 28]. For simplicity, let $\left(u_{1}(t, x), v_{1}(t, x)\right)>\left(u_{2}(t, x), v_{2}(t, x)\right)$ denote $u_{1}(t, x)>$ $u_{2}(t, x)$ and $v_{1}(t, x)>v_{2}(t, x)$.

Lemma 4 (see [29]). Assume that $u(t, x)$ is defined by

$$
\begin{gathered}
u_{t}-d_{1} \Delta u=r u\left(1-\frac{u}{K}\right), \quad x \in \Omega, t>0, \\
\frac{\partial u}{\partial n}=0, \quad x \in \partial \Omega, \\
u(x, 0)=u_{0}(x)>0, \quad x \in \Omega .
\end{gathered}
$$

Then $\lim _{t \rightarrow \infty} u(x, t)=K$.

Theorem 5. If $r-(m / 2 \sqrt{a} h) K>0$, then for system (3), the positive equilibrium $\left(u^{*}, v^{*}\right)$ is globally asymptotically stable.

Proof. From the maximum principle of parabolic equations, it is known that for any initial value $\left(u_{0}(t, x), v_{0}(t, x)\right)>(0,0)$, the corresponding nonnegative solution $(u(t, x), v(t, x))$ is strictly positive for $t>0$. Because $r-(m / 2 \sqrt{a} h) K>0$, it is possible to choose $\varepsilon_{0}$ satisfying

$$
0<\varepsilon_{0}<\left(r-\frac{m}{2 \sqrt{a} h} K\right)\left[\frac{m}{2 \sqrt{a} h}+\frac{m}{2 \sqrt{a}}+\frac{r}{K}(1+h)\right]^{-1} .
$$

Because

$$
\frac{\partial u}{\partial t}-d_{1} \Delta u=r u\left(1-\frac{u}{K}\right)-\frac{m u^{2} v\left(t-\tau_{1}\right)}{a+u^{2}} \leq r u\left(1-\frac{u}{K}\right)
$$

according to Lemma 4 and the comparison principle of parabolic equations, there exists $t_{1}>0$ such that, for any $t>t_{1}, u(x, t) \leq K+\varepsilon_{0} \triangleq \bar{u}$. This in turn implies that

$$
\frac{\partial v}{\partial t}-d_{1} \Delta v=s v\left(1-\frac{h v}{u\left(t-\tau_{2}\right)}\right) \leq s v\left(1-\frac{h v}{K+\varepsilon_{0}}\right)
$$

for $t>t_{1}+\tau_{2}$.

Hence there exists $t_{2}>t_{1}$ such that, for any $t>t_{2}$,

$$
v(x, t) \leq \frac{K+\varepsilon_{0}}{h}+\varepsilon_{0} \triangleq \bar{v} .
$$

Consequently,

$$
\begin{aligned}
\frac{\partial u}{\partial t}-d_{1} \Delta u & =r u\left(1-\frac{u}{K}\right)-\frac{m u^{2} v\left(t-\tau_{1}\right)}{a+u^{2}} \\
& \geq r u\left(1-\frac{u}{K}\right)-\frac{m u^{2} v\left(t-\tau_{1}\right)}{2 \sqrt{a} u} \\
& \geq r u\left(1-\frac{u}{K}\right)-\frac{m\left(K+(h+1) \varepsilon_{0}\right)}{2 h \sqrt{a}} u
\end{aligned}
$$

for $t>t_{2}+\tau_{1}$.

Because $r-(m / 2 \sqrt{a} h) K>0$, it can be easily verified that

$$
\begin{gathered}
K\left(1-\frac{m\left(K+(h+1) \varepsilon_{0}\right)}{2 r h \sqrt{a}}\right)>0, \\
K\left(1-\frac{m\left(K+(h+1) \varepsilon_{0}\right)}{2 r h \sqrt{a}}\right)-\varepsilon_{0}>0 .
\end{gathered}
$$

Hence, there exists $t_{3}>t_{2}$ such that, for any $t>t_{3}$,

$$
u(x, t) \geq K\left(1-\frac{m\left(K+(h+1) \varepsilon_{0}\right)}{2 r h \sqrt{a}}\right)-\varepsilon_{0} \triangleq \underline{u} .
$$

This implies that

$$
\frac{\partial v}{\partial t}-d_{1} \Delta v=s v\left(1-\frac{h v}{u\left(t-\tau_{2}\right)}\right) \geq s v-\frac{s h v^{2}}{\underline{u}}
$$

for $t>t_{3}+\tau_{2}$. Again it can be verified that

$$
\frac{u}{\bar{h}}-\varepsilon_{0}=\frac{K}{h}\left(1-\frac{m\left(K+(h+1) \varepsilon_{0}\right)}{2 r h \sqrt{a}}\right)-\frac{\varepsilon_{0}}{h}-\varepsilon_{0}>0,
$$


and hence there exists $t_{4}>t_{3}$ such that, for any $t>t_{4}$,

$$
v(x, t) \geq \frac{K}{h}\left(1-\frac{m\left(K+(h+1) \varepsilon_{0}\right)}{2 r h \sqrt{a}}\right)-\frac{\varepsilon_{0}}{h}-\varepsilon_{0} \triangleq \underline{v} .
$$

Therefore, for $t>t_{4}$, it is possible to obtain

$$
\underline{u} \leq u(x, t) \leq \bar{u}, \quad \underline{v} \leq v(x, t) \leq \bar{v}
$$

and $\underline{u}, \bar{u}, \underline{v}, \bar{v}$ satisfy

$$
\begin{array}{ll}
0 \geq r\left(1-\frac{\bar{u}}{K}\right)-\frac{m \bar{u} \underline{v}}{a+\bar{u}^{2}}, & 0 \geq s\left(1-\frac{h \bar{v}}{\bar{u}}\right), \\
0 \leq r\left(1-\frac{\underline{u}}{K}\right)-\frac{m \underline{u} \bar{v}}{a+\underline{u}^{2}}, & 0 \leq s\left(1-\frac{h \underline{v}}{\underline{u}}\right) .
\end{array}
$$

This implies that $(\bar{u}, \bar{v})$ and $(\underline{u}, \underline{v})$ are a pair of coupled upper and lower solutions of system (3), as in the definition in [29], for the reason that (3) is a mixed quasimonotonic system.

It is clear that there exists $K>0$ such that, for any $(\underline{u}, \underline{v}) \leq$ $\left(u_{1}, v_{1}\right),\left(u_{2}, v_{2}\right) \leq(\bar{u}, \bar{v})$,

$$
\begin{aligned}
& \left|r u_{1}\left(1-\frac{u_{1}}{K}\right)-\frac{m u_{1}^{2} v_{1}}{a+u_{1}^{2}}-r u_{2}\left(1-\frac{u_{2}}{K}\right)+\frac{m u_{2}^{2} v_{2}}{a+u_{2}^{2}}\right| \\
& \quad \leq K\left(\left|u_{1}-u_{2}\right|+\left|v_{1}-v_{2}\right|\right), \\
& \left|s v_{1}\left(1-\frac{h v_{1}}{u_{1}}\right)-s v_{2}\left(1-\frac{h v_{2}}{u_{2}}\right)\right| \\
& \quad \leq K\left(\left|u_{1}-u_{2}\right|+\left|v_{1}-v_{2}\right|\right) .
\end{aligned}
$$

Define two iteration sequences $\left(\bar{u}^{(m)}, \bar{v}^{(m)}\right)$ and $\left(\underline{u}^{(m)}, \underline{v}^{(m)}\right)$ as follows: for $m \geq 1$,

$$
\begin{aligned}
& \bar{u}^{(m)}= \bar{u}^{(m-1)}+\frac{1}{K} \bar{u}^{(m-1)} \\
& \times\left(r\left(1-\frac{\bar{u}^{(m-1)}}{K}\right)-\frac{m \bar{u}^{(m-1)} \underline{v}^{(m-1)}}{a+\bar{u}^{(m-1)^{2}}}\right), \\
& \bar{v}^{(m)}=\bar{v}^{(m-1)}+\frac{1}{K} s \bar{v}^{(m-1)}\left(1-\frac{h \bar{v}^{(m)}}{\bar{u}^{(m)}}\right), \\
& \underline{u}^{(m)}=\underline{u}^{(m-1)}+\frac{1}{K} \underline{u}^{(m-1)} \\
& \quad \times\left(r\left(1-\frac{\underline{u}^{(m-1)}}{K}\right)-\frac{m \underline{u}^{(m-1)} \bar{v}^{(m-1)}}{a+\underline{u}^{(m-1)}}\right), \\
& \underline{v}^{(m)}=\underline{v}^{(m-1)}+\frac{1}{K} s \underline{v}^{(m-1)}\left(1-\frac{h \underline{v}^{(m-1)}}{\underline{u}^{(m-1)}}\right),
\end{aligned}
$$

where $\left(\bar{u}^{(0)}, \bar{v}^{(0)}\right)=(\bar{u}, \bar{v}),\left(\underline{u}^{(0)}, \underline{v}^{(0)}\right)=(\underline{u}, \underline{v})$.

Then, for $m \geq 1$,

$$
\begin{aligned}
(\underline{u}, \underline{v}) & \leq\left(\underline{u}^{(m)}, \underline{v}^{(m)}\right) \leq\left(\underline{u}^{(m+1)}, \underline{v}^{(m+1)}\right) \\
& \leq\left(\bar{u}^{(m+1)}, \bar{v}^{(m+1)}\right) \leq\left(\bar{u}^{(m)}, \bar{v}^{(m)}\right) \leq(\bar{u}, \bar{v}),
\end{aligned}
$$

and there exist $(\widetilde{u}, \widetilde{v})>(0,0)$ and $(\widehat{u}, \widehat{v})>(0,0)$ such that $\lim _{m \rightarrow \infty} \bar{u}^{(m)}=\widetilde{u}, \lim _{m \rightarrow \infty} \bar{v}^{(m)}=\widetilde{v}, \lim _{m \rightarrow \infty} \underline{u}^{(m)}=\widehat{u}$, $\lim _{m \rightarrow \infty} \underline{v}^{(m)}=\widehat{v}$, and

$$
\begin{array}{ll}
0=\widetilde{u}\left(r\left(1-\frac{\widetilde{u}}{K}\right)-\frac{m \tilde{u} \widehat{v}}{a+\widetilde{u}^{2}}\right), & 0=\widetilde{v}\left(s\left(1-\frac{h \widetilde{v}}{\widetilde{u}}\right)\right), \\
0=\widehat{u}\left(r\left(1-\frac{\widehat{u}}{K}\right)-\frac{m \widehat{u} \widetilde{v}}{a+\widehat{u}^{2}}\right), & 0=\widehat{v}\left(s\left(1-\frac{h \widehat{v}}{\widehat{u}}\right)\right) .
\end{array}
$$

Because $\left(u^{*}, v^{*}\right)$ is the unique positive constant equilibrium of system (3), it must hold that $(\widetilde{u}, \widetilde{v})=(\widehat{u}, \widehat{v})=\left(u^{*}, v^{*}\right)$.

According to the results of $[27,28]$, the solution $(u(x, t), v(x, t))$ of system (3) satisfies

$$
\begin{array}{r}
\lim _{t \rightarrow \infty} u(x, t)=u^{*}, \quad \lim _{t \rightarrow \infty} v(x, t)=v^{*} \\
\text { uniformly for } x \in \bar{\Omega} .
\end{array}
$$

Hence, the constant equilibrium $\left(u^{*}, v^{*}\right)$ is globally asymptotically stable.

\section{Direction and Stability of Hopf Bifurcation}

Part Two has already shown that system (3) undergoes Hopf bifurcation at the interior equilibrium $\left(u^{*}, v^{*}\right)$ for $\tau_{1}+\tau_{2}=$ $\tau_{k}, k=0,1,2, \ldots$. In this section, the direction, stability, and period of the periodic solutions from the steady state will be studied by applying the method introduced by Hassard et al. [30] and the center manifold theorem due to [31-35].

For convenience, let $u^{\prime}=u\left(t-\tau_{2}\right), v^{\prime}=v(t), \tau=\tau_{1}+\tau_{2}$. Then system (3) is equal to a single-delay system:

$$
\begin{gathered}
\frac{\partial u^{\prime}}{\partial t}=d_{1} \Delta u^{\prime}+r u\left(1-\frac{u^{\prime}}{K}\right)-\frac{m u^{\prime 2} v^{\prime}(t-\tau)}{a+u^{\prime 2}}, \\
x \in(0, l \pi), \quad t>0, \\
\frac{\partial v^{\prime}}{\partial t}=d_{1} \Delta v^{\prime}+v^{\prime}\left[s\left(1-\frac{h v^{\prime}}{u^{\prime}}\right)\right], \\
x \in(0, l \pi), \quad t>0, \\
\frac{\partial u^{\prime}}{\partial n}=\frac{\partial v^{\prime}}{\partial n}=0, \quad x=0, l \pi, t>0 .
\end{gathered}
$$

Let $x(t)=u^{\prime}(\tau t)-u^{*}, y(t)=v^{\prime}(\tau t)-v^{*}, \tau=\tau_{0}+\mu$. Then $\mu=0$ is the Hopf bifurcation value of system (48), which can be written as follows:

$$
\frac{\partial U(t)}{\partial t}=\tau_{0} d \Delta U(t)+\tau_{0} L\left(U_{t}\right)+G\left(U_{t}\right)
$$


where

$L(\phi)$

$$
\left.\begin{array}{c}
=\left(\left[r-\frac{2 r u^{*}}{K}-\frac{2 a m u^{*} v^{*}}{\left(a+u^{* 2}\right)^{2}}\right] \phi_{1}(0)-\frac{m u^{*}}{a+u^{* 2}} \phi_{2}(-1)\right. \\
\frac{s}{h} \phi_{1}(0)-s \phi_{2}(0)
\end{array}\right),
$$

with

$$
\begin{aligned}
F_{1}(\phi)= & {\left[r\left(\phi_{1}(0)+u^{*}\right)\left(1-\frac{\phi_{1}(0)+u^{*}}{K}\right)\right.} \\
& \left.-\frac{m\left(\phi_{1}(0)+u^{*}\right)^{2}\left(\phi_{2}(-1)+v^{*}\right)}{a+\phi_{1}(0)+u^{* 2}}\right] \phi_{1}(0) \\
& -\frac{m u^{*}}{a+u^{* 2}} \phi_{2}(-1), \\
F_{2}(\phi)= & s\left(\phi_{2}(0)+v^{*}\right)\left(1-\frac{h\left(\phi_{2}(0)+v^{*}\right)}{\phi_{1}(0)+u^{*}}\right) \\
& -\frac{s}{h} \phi_{1}(0)+s \phi_{2}(0)
\end{aligned}
$$

for $\phi \in C([-1,0], X)$.

From the previous discussion, it is known that $\pm i w_{0}$ is a pair of simple purely imaginary eigenvalues of the linear system (7) and the following linear functional differential equation:

$$
\frac{d z(t)}{d t}=\tau_{0} L(z t)
$$

By the Riesz representation theorem, there exists a $2 \times 2$ matrix function $\eta(\theta, \mu)(\theta \in[-1,0])$, whose elements are bounded variables, such that

$$
\begin{array}{r}
\left(\tau_{0}+\mu\right) L(\phi)=\int_{-1}^{0} d \eta(\theta, \mu) \phi(\theta) \\
\text { for } \phi \in C\left([-1,0], R^{2}\right) .
\end{array}
$$

In fact, it is possible to choose

$$
\begin{aligned}
\eta(\theta, \mu) & \left(\tau_{0}+\mu\right)\left(\left[\begin{array}{cc}
r-\frac{2 r u^{*}}{K}-\frac{2 a m u^{*} v^{*}}{\left(a+u^{* 2}\right)^{2}} & 0 \\
\frac{s}{h} & -s
\end{array}\right) \delta(\theta)\right. \\
& \left.+\left(\begin{array}{cc}
0 & -\frac{m u^{*}}{a+u^{* 2}} \\
0 & 0
\end{array}\right) \delta(\theta+1)\right)
\end{aligned}
$$

where $\delta(\theta)$ is a Dirac delta function satisfying

$$
\delta(\theta)= \begin{cases}1, & \theta=0 \\ 0, & \theta \neq 0 .\end{cases}
$$

For $\phi \in C^{1}\left([-1,0], R^{2}\right)$, define $A(0)$ as

$$
\begin{gathered}
A(0) \phi(\theta)= \begin{cases}\frac{d \phi(\theta)}{d \theta}, & \theta \in[-1,0), \\
\int_{-1}^{0} d \eta(\theta, 0) \phi(\theta), & \theta=0,\end{cases} \\
R(0) \phi(\theta)= \begin{cases}0, & \theta \in[-1,0), \\
F(\phi, \mu), & \theta=0 .\end{cases}
\end{gathered}
$$

For $\psi \in C^{1}\left([-1,0],\left(R^{2}\right)^{*}\right)$, define

$$
A^{*}(\psi(s))= \begin{cases}-\frac{d \psi(s)}{d s}, & s \in(0,1], \\ \int_{-1}^{0} d \eta(\theta, 0) \psi(-\xi), & s=0 .\end{cases}
$$

Then $A(0)$ and $A^{*}$ are adjoint operators under the bilinear form:

$$
\begin{aligned}
(\psi(s), \phi(\theta))_{0}= & \bar{\psi}(0) \phi(0) \\
& -\int_{-1}^{0} \int_{0}^{\theta} \bar{\psi}(\xi-\theta) d \eta(0, \theta) \phi(\xi) d \xi .
\end{aligned}
$$

Therefore, $\pm i w_{0}$ are eigenvalues of $A(0)$ as well as $A^{*}$. Next, the eigenvectors of $A(0)$ and $A^{*}$ corresponding to the eigenvalues $i w_{0}$ and $-i w_{0}$ can be calculated. Let

$$
q(\theta)=\left(\begin{array}{l}
1 \\
C
\end{array}\right) e^{i w_{0} \tau_{0} \theta} .
$$

Under the condition $A(0) q(0)=i w_{0} \tau_{0} q(0)$, that is,

$$
\begin{aligned}
& \tau_{0}\left(\begin{array}{cc}
r-\frac{2 r u^{*}}{K}-\frac{2 a m u^{*} v^{*}}{\left(a+u^{* 2}\right)^{2}}-i w_{0} & -\frac{m u^{*}}{a+u^{* 2}} e^{-i w_{0} \tau_{0}} \\
\frac{s}{h} & -s-i w_{0}
\end{array}\right)\left(\begin{array}{l}
1 \\
C
\end{array}\right) \\
& =\left(\begin{array}{l}
0 \\
0
\end{array}\right),
\end{aligned}
$$

it follows that

$$
C=\frac{s}{h\left(s+i w_{0}\right)} \text {. }
$$

Similarly, let $q^{*}(s)=E(1 \quad D) e^{i w_{0} \tau_{0} s}$, and with $A^{*} q^{*}(0)=$ $-i w_{0} \tau_{0} q^{*}(0)$, that is,

$$
\begin{aligned}
& \tau_{0}\left(\begin{array}{cc}
r-\frac{2 r u^{*}}{K}-\frac{2 a m u^{*} v^{*}}{\left(a+u^{* 2}\right)^{2}}+i w_{0} & \frac{s}{h} \\
-\frac{m u^{*}}{a+u^{* 2}} e^{-i w_{0} \tau_{0}} & -s+i w_{0}
\end{array}\right)\left(\begin{array}{c}
1 \\
D
\end{array}\right) \\
& =\left(\begin{array}{l}
0 \\
0
\end{array}\right),
\end{aligned}
$$


it is possible to obtain

$$
D=\frac{-h\left(r-2 r u^{*} / K-2 a m u^{*} v^{*} /\left(a+u^{* 2}\right)^{2}+i w_{0}\right)}{s} .
$$

According to the conditions $\left(q^{*}, q\right)=1$ and $\left(q^{*}, \bar{q}\right)=0$,

$$
\begin{aligned}
& \left(q^{*}, q\right)=\overline{q^{*}}(0) q(0)-\int_{-1}^{0} \int_{\xi=0}^{\theta} \overline{q^{*}}(\xi-\theta) d \eta(0, \theta) q(\xi) d \xi \\
& =\bar{E}\left(\begin{array}{ll}
1 & \bar{D}
\end{array}\right)\left(\begin{array}{l}
1 \\
C
\end{array}\right)-\int_{-1}^{0} \int_{\xi=0}^{\theta} \bar{E}(1 \bar{D}) e^{-i w_{0} \tau_{k}(\xi-\theta)} d \eta(\theta) \\
& \times\left(\begin{array}{c}
1 \\
C
\end{array}\right) e^{i w_{0} \tau_{k} \xi} d \xi \\
& =\bar{E}\left\{1+\bar{D} C-\int_{-1}^{0} \theta(1 \bar{D}) d \eta(0, \theta)\left(\begin{array}{l}
1 \\
C
\end{array}\right) e^{i w_{0} \tau_{k} \theta}\right\} \\
& =\bar{E}\left\{1+\bar{D} C+\left(\begin{array}{ll}
1 & \bar{D}
\end{array}\right) \tau_{0}\left(\begin{array}{cc}
0 & -\frac{m u^{*}}{a+u^{* 2}} \\
0 & 0
\end{array}\right)\right. \\
& \left.\times\left(\begin{array}{c}
1 \\
C
\end{array}\right) e^{-i w_{0} \tau_{k}}\right\} \\
& =\bar{E}\left\{1+\bar{D} C+\tau_{0} C\left(-\frac{m u^{*}}{a+u^{* 2}}\right) e^{-i w_{0} \tau_{k}}\right\} \text {. }
\end{aligned}
$$

Therefore,

$$
E=\frac{1}{1+D \bar{C}+\tau_{0} \bar{C}\left(-m u^{*} /\left(a+u^{* 2}\right)\right) e^{i w_{0} \tau_{k}}} .
$$

Let $\Phi=(q(\theta), \bar{q}(\theta)), \Psi=\left(q^{*}(s), \bar{q}^{*}(s)\right)^{T}, I=\left(\begin{array}{cc}1 & 0 \\ 0 & 1\end{array}\right)$; then

$$
(\Psi, \Phi)_{0}=I \text {. }
$$

Therefore, the center subspace of system (52) is $P=$ $\operatorname{span}\{q(\theta), \bar{q}(\theta)\}$, and the adjoint subspace is $P^{*}=\operatorname{span}\left\{q^{*}(s)\right.$, $\left.\bar{q}^{*}(s)\right\}$. Let $f_{0}=\left(f_{0}^{1}, f_{0}^{2}\right)$, where

$$
f_{0}^{1}=\left(\begin{array}{l}
1 \\
0
\end{array}\right), \quad f_{0}^{2}=\left(\begin{array}{l}
0 \\
1
\end{array}\right) .
$$

Using the notion from [30], it is also possible to define

$$
c \cdot f_{0}=c_{1} f_{0}^{1}+c_{2} f_{0}^{2}
$$

for $c=\left(c_{1}, c_{2}\right)^{T} \in C^{2}$

Define $\left(\psi \cdot f_{0}\right)(\theta)=\psi(\theta) \cdot f_{0}$ for $\psi(\theta) \in[-1,0]$ and

$$
\langle u, v\rangle=\frac{1}{l \pi} \int_{0}^{l \pi} u_{1} \bar{v}_{1} d x+\frac{1}{l \pi} \int_{0}^{l \pi} u_{2} \bar{v}_{2} d x
$$

for $u=\left(u_{1}, u_{2}\right), v=\left(v_{1}, v_{2}\right) \in C\left([0, l \pi], R^{2}\right)$.

Hence,

$$
\left\langle\phi, f_{0}\right\rangle=\left(\left\langle\phi, f_{0}^{1}\right\rangle,\left\langle\phi, f_{0}^{2}\right\rangle\right)^{T}
$$

for $\phi \in C([-1,0], X)$. Then the center subspace of linear system (7) is given by $P_{C N} \mathrm{C}$, where

$$
\begin{gathered}
P_{C N} \phi=\Phi\left(\Psi,\left\langle\phi, f_{0}\right\rangle\right)_{0} \cdot f_{0}, \quad \phi \in C, \\
P_{C N} \mathrm{C}=\left\{(q(\theta) z+\bar{q}(\theta) \bar{z}) \cdot f_{0}: z \in C\right\},
\end{gathered}
$$

and $\mathrm{C}=P_{\mathrm{CN}} \mathrm{C} \oplus P_{S} \mathrm{C}$, where $P_{S} \mathrm{C}$ is a stable subspace.

According to [30], it is known that the infinitesimal generator $A_{U}$ of linear system (7) satisfies

$$
A_{U} \psi=\dot{\psi}(\theta) \text {. }
$$

Moreover, $\psi \in \operatorname{dom}\left(A_{U}\right)$ if and only if

$$
\begin{aligned}
& \dot{\psi}(\theta) \in \mathrm{C}, \quad \psi(0) \in \operatorname{dom}(\Delta), \\
& \dot{\psi}(\theta)(0)=\tau_{0} \Delta \psi(0)+\tau_{0} L_{0}(\psi) .
\end{aligned}
$$

Setting $\mu=0$ in (49), the center manifold

$$
W(z, \bar{z})=W_{20}(\theta) \frac{z^{2}}{2}+W_{11}(\theta) z \bar{z}+W_{02}(\theta) \frac{\bar{z}^{2}}{2}+\cdots
$$

can be obtained in $P_{S} C$. The flow of system (49) can be written as

$$
u_{t}=\Phi(z(t), \bar{z}(t))^{T} \cdot f_{0}+W(z(t), \bar{z}(t))
$$

where

$$
\begin{aligned}
\dot{z}(t)= & i w_{0} \tau_{0} z(t)+\overline{q^{*}}(0) \\
& \times\left\langle G\left(\Phi(z(t), \bar{z}(t))^{T} \cdot f_{0}+W(z, \bar{z}), 0\right), f_{0}\right\rangle \\
\triangleq & i w_{0} \tau_{0} z(t)+g(z, \bar{z}),
\end{aligned}
$$

$$
\begin{aligned}
g(z, \bar{z})= & \overline{q^{*}}(0) \\
& \times\left\langle G\left(\Phi(z(t), \bar{z}(t))^{T} \cdot f_{0}+W(z, \bar{z}), 0\right), f_{0}\right\rangle \\
= & g_{20}(\theta) \frac{z^{2}}{2}+g_{11}(\theta) z \bar{z}+g_{02}(\theta) \frac{\bar{z}^{2}}{2} \\
& +g_{21}(\theta) \frac{z^{2} \bar{z}}{2}+\cdots .
\end{aligned}
$$

From Taylor's formula,

$$
\begin{aligned}
f(u, v)= & \frac{m\left(u+u^{*}\right)^{2}\left(v+v^{*}\right)}{a+\left(u+u^{*}\right)^{2}} \\
= & \frac{m u^{* 2} v^{*}}{a+u^{* 2}}+\frac{2 a m u^{*} v^{*}}{\left(a+u^{*}\right)^{2}} u+\frac{m u^{* 2}}{a+u^{* 2}} v+b_{20} u^{2} \\
& +b_{11} u v+b_{30} u^{3}+b_{21} u^{2} v+O(4),
\end{aligned}
$$




$$
\begin{aligned}
g(u, v)= & \frac{s h\left(v+v^{*}\right)^{2}}{u+u^{*}} \\
= & \operatorname{sh}\left(\frac{v^{*}}{h}-\frac{1}{h^{2}} u+\frac{2}{h} v+\frac{1}{h^{2} u^{*}} u^{2}+\frac{1}{u^{*}} v^{2}-\frac{2}{h u^{*}} u v\right. \\
& \left.\quad+\frac{2}{h^{2} u^{* 2}} u^{2} v-\frac{1}{h^{2} u^{* 2}} u^{3}+O(4)\right),
\end{aligned}
$$

where

$$
\begin{aligned}
b_{11}= & \frac{2 a m u^{*}}{\left(a+u^{*}\right)^{2}}, \\
b_{20}= & \frac{a m v^{*}\left(a^{2}+2 a u^{* 2}+u^{* 4}\right)-4 a m u^{* 2} v^{*}\left(a+u^{* 2}\right)}{\left(a+u^{* 2}\right)^{4}}, \\
b_{21}= & \frac{a m\left(a+u^{* 2}\right)-4 a m u^{* 2}\left(a+u^{* 2}\right)}{\left(a+u^{* 2}\right)^{3}}, \\
b_{30}= & {\left[-8 a m u^{*} v^{*}\left(a+u^{* 2}\right)-8 a m v^{*}\right.} \\
& \left.\times\left(3 u^{* 2}-2 a u^{*}\right)+64 a m u^{* 3} v^{*}\right] \\
& \times\left(a+u^{* 2}\right)\left(\left(a+u^{*}\right)^{2}\right)^{-1}, \\
O(4)= & O\left(\|(u, v)\|^{4}\right) .
\end{aligned}
$$

Let $G(\phi, 0)=\tau_{0}\left(G_{1}, G_{2}\right)^{T}$, where

$$
\begin{aligned}
G_{1}= & \left(-\frac{r}{K}-b_{20}\right) \phi_{1}^{2}(0)-b_{11} \phi_{1}(0) \phi_{2}(-1) \\
& -b_{30} \phi_{1}^{3}(0)-b_{21} \phi_{1}^{2}(0) \phi_{2}(-1)+O(4), \\
G_{2}= & -\frac{s}{h u^{*}} \phi_{1}^{2}(0)-\frac{s h}{u^{*}} \phi_{2}^{2}(0)+\frac{2 s}{u^{*}} \phi_{1}(0) \phi_{2}(0) \\
& -\frac{2 s}{h u^{* 2}} \phi_{1}^{2}(0) \phi_{2}(0)+\frac{s}{h u^{* 2}} \phi_{1}^{3}(0)+O(4) .
\end{aligned}
$$

From (77),

$$
\begin{aligned}
g_{20}= & 2 \bar{E} \tau_{0}\left[\left(-\frac{r}{K}-b_{20}\right)-b_{11} C e^{-i w_{0} \tau_{0}}\right] \\
& +2 \bar{E} \tau_{0} \bar{D}\left[-\frac{s}{h u^{*}}-\frac{s h}{u^{*}} C^{2}+\frac{2 s}{u^{*}} C\right], \\
g_{11}= & \bar{E} \tau_{0}\left[2\left(-\frac{r}{K}-b_{20}\right)-b_{11}\left(C e^{-i w_{0} \tau_{0}}+\bar{C} e^{i w_{0} \tau_{0}}\right)\right] \\
& +\bar{E} \tau_{0} \bar{D}\left[-\frac{2 s}{h u^{*}}-\frac{2 s h}{u^{*}} C \bar{C}+\frac{2 s}{u^{*}}(C+\bar{C})\right], \\
g_{02}= & 2 \bar{E} \tau_{0}\left[\left(-\frac{r}{K}-b_{20}\right)-b_{11} \bar{C} e^{i w_{0} \tau_{0}}\right] \\
& +2 \bar{E} \tau_{0} \bar{D}\left[-\frac{s}{h u^{*}}-\frac{s h}{u^{*}} \bar{C}^{2}+\frac{2 s}{u^{*}} \bar{C}\right],
\end{aligned}
$$

$$
\begin{aligned}
g_{21}= & 2 \bar{E} \tau_{0}\left[\left(-\frac{r}{K}-b_{20}\right)-\bar{D} \frac{s}{h u^{*}}\right] \\
& \times \frac{1}{l \pi} \int_{0}^{l \pi}\left(W_{20}^{(1)}(0)+2 W_{11}^{(1)}(0)\right) d x \\
& -2 \bar{E} \tau_{0} \bar{D} \frac{s h}{u^{*}} \frac{1}{l \pi} \int_{0}^{l \pi}\left(W_{20}^{(1)}(0) \bar{C}+2 W_{11}^{(1)}(0) C\right) d x \\
& -E \tau_{0} b_{11} \frac{1}{l \pi} \int_{0}^{l \pi}\left(W_{20}^{(1)}(0) \bar{C} e^{i w_{0} \tau_{0}}+2 W_{11}^{(1)}(0) C e^{-i w_{0} \tau_{0}}\right. \\
& +\bar{E} \tau_{0} \bar{D} \frac{2 s}{u^{*}} \frac{1}{l \pi} \int_{0}^{l \pi}\left(W_{20}^{(1)}(0) \bar{C}+2 W_{11}^{(1)}(0) C\right. \\
& -2 \bar{E} \tau_{0} b_{21}\left(\bar{C} e^{i w_{0} \tau_{0}}+2 C e^{-i w_{0} \tau_{0}}\right) \\
& -2 \bar{E} \tau_{0} \bar{D} \frac{2 s}{h u^{* 2}}(\bar{C}+2 C) \\
& -6 \bar{E} \tau_{0} b_{30}+6 \bar{E} \tau_{0} \bar{D} \frac{s}{h u^{* 2}} . \\
& \left.+W_{20}^{(2)}(0)+2 W_{11}^{(2)}(0)\right) d x \\
& \\
& \\
& \\
&
\end{aligned}
$$

In the last formula, $W_{20}(0), W_{20}(-1), W_{11}(0)$, and $W_{11}(-1)$ are still unknown. Therefore, it is necessary to compute $W_{20}(\theta)$ and $W_{11}(\theta)$, as described below.

From (74), it is possible to obtain

$$
\begin{gathered}
\dot{W}=W_{20} z \dot{z}+W_{11} \bar{z} \dot{z}+W_{11} z \dot{\bar{z}}+W_{02} z \dot{\bar{z}}+\cdots, \\
A_{U} W=A_{U} W_{20} \frac{z^{2}}{2}+A_{U} W_{11} z \bar{z}+W_{02} A_{U} \frac{\bar{z}^{2}}{2}+\cdots .
\end{gathered}
$$

In addition, $W(z, \bar{z})$ satisfies

$$
\begin{aligned}
\dot{W}= & A_{U} W+X_{0} G\left(\Phi(z, \bar{z})^{T} \cdot f_{0}+W(z, \bar{z}), 0\right) \\
& -\Phi\left(\Psi,\left\langle X_{0} G\left(\Phi(z, \bar{z})^{T} \cdot f_{0}+W(z, \bar{z}), 0\right), f_{0}\right\rangle\right)_{0} \cdot f_{0} \\
\triangleq & A_{U} W+H_{20} \frac{z^{2}}{2}+H_{11} z \bar{z}+H_{02} \frac{\bar{z}^{2}}{2}+\cdots .
\end{aligned}
$$

Therefore,

$$
\begin{gathered}
2 i w_{0} \tau_{0} W_{20}=A_{U} W_{20}+H_{20}, \\
-A_{U} W_{11}=H_{11}, \\
-2 i w_{0} \tau_{0} W_{02}=A_{U} W_{02}+H_{02} .
\end{gathered}
$$

For $-1 \leq \theta<0$,

$$
\begin{aligned}
& H_{20} \frac{z^{2}}{2}+H_{11} z \bar{z}+H_{02} \frac{\bar{z}^{2}}{2}+\cdots \\
& \quad=-\Phi\left(\Psi,\left\langle X_{0} G\left(\Phi(z, \bar{z})^{T} \cdot f_{0}+W(z, \bar{z}), 0\right), f_{0}\right\rangle\right)_{0} \cdot f_{0},
\end{aligned}
$$


and therefore

$$
\begin{aligned}
& H_{20}(\theta)=-\left[g_{20} q(\theta)+\bar{g}_{02} \bar{q}(\theta)\right] \cdot f_{0}, \\
& H_{11}(\theta)=-\left[g_{11} q(\theta)+\bar{g}_{11} \bar{q}(\theta)\right] \cdot f_{0} .
\end{aligned}
$$

From (84) and (86),

$$
\dot{W}_{20}(\theta)=2 i w_{0} \tau_{0} W_{20}(\theta)+\left[g_{20} q(\theta)+\bar{g}_{02} \bar{q}(\theta)\right] \cdot f_{0} .
$$

Solving for $W_{20}(\theta)$,

$$
W_{20}(\theta)=\frac{i g_{20} q(\theta)}{w_{0} \tau_{0}} \cdot f_{0}+\frac{i \bar{g}_{02} \bar{q}(\theta)}{3 w_{0} \tau_{0}} \cdot f_{0}+E_{1} e^{2 i w_{p} \tau_{0} \theta}
$$

Similarly,

$$
W_{11}(\theta)=\frac{-i g_{11} q(\theta)}{w_{0} \tau_{0}} \cdot f_{0}+\frac{i \bar{g}_{11} \bar{q}(\theta)}{w_{0} \tau_{0}} \cdot f_{0}+E_{2}
$$

where $E_{1}$ and $E_{2}$ are both two-dimensional vectors and can be determined as follows.

For $\theta=0$, according to the definition of $A_{U}$ and the first two equations of (84),

$$
\begin{aligned}
2 i w_{0} \tau_{0} W_{20}(0)= & \int_{-1}^{0} d \eta(\theta, 0) W_{20}(\theta)+H_{20}(0) \\
& -\int_{-1}^{0} d \eta(\theta, 0) W_{11}(\theta)=H_{11}(0) .
\end{aligned}
$$

Then

$$
\begin{aligned}
H_{20}(0)= & -\left[g_{20} q(0)+\bar{g}_{02} \bar{q}(0)\right] \cdot f_{0}+2 \tau_{0} \\
& \times\left(\begin{array}{c}
-\frac{r}{K}-b_{20}-b_{11} C e^{-i w_{0} \tau_{0}} \\
-\frac{s}{h u^{*}}-\frac{s h}{u^{*}} C^{2}+\frac{2 s}{u^{*}} C
\end{array}\right) .
\end{aligned}
$$

Note that

$$
\begin{gathered}
\int_{-1}^{0} d \eta(\theta, 0) e^{i w_{0} \tau_{0} \theta} q(0)=i w_{0} \tau_{0} q(0), \\
\int_{-1}^{0} d \eta(\theta, 0) e^{-i w_{0} \tau_{0} \theta} \bar{q}(0)=-i w_{0} \tau_{0} \bar{q}(0) .
\end{gathered}
$$

It follows that

$$
\begin{aligned}
& 2 \tau_{0}\left(\begin{array}{c}
-\frac{r}{K}-b_{20}-b_{11} C e^{-i w_{0} \tau_{0}} \\
-\frac{s}{h u^{*}}-\frac{s h}{u^{*}} C^{2}+\frac{2 s}{u^{*}} C
\end{array}\right) \\
& =g_{20} q(0)+\bar{g}_{02} \bar{q}(0)+2 i w_{0} \tau_{0} W_{20}(0) \\
& -\int_{-1}^{0} d \eta(\theta, 0) W_{20}(\theta) \\
& =-g_{20} q(0)+\frac{\bar{g}_{02} \bar{q}(0)}{3}+2 i w_{0} \tau_{0} E_{1} \\
& -\int_{-1}^{0} d \eta(\theta, 0)\left[\frac{i g_{20} q(\theta)}{w_{0} \tau_{0}}+\frac{i \bar{g}_{02} \bar{q}(\theta)}{3 w_{0} \tau_{0}}+E_{1} e^{2 i w_{p} \tau_{0} \theta}\right] \\
& =\left(2 i w_{0} \tau_{0} I-\int_{-1}^{0} d \eta(\theta, 0) e^{2 i w_{p} \tau_{0} \theta}\right) E_{1} \\
& =\left[2 i w_{0} \tau_{0}\left(\begin{array}{ll}
1 & 0 \\
0 & 1
\end{array}\right)-\tau_{0}\right. \\
& \left.\times\left(\begin{array}{cc}
r-\frac{2 r u^{*}}{K}-\frac{2 a m u^{*} v^{*}}{\left(a+u^{* 2}\right)^{2}} & -\frac{m u^{*}}{a+u^{* 2}} e^{-2 i w_{0} \tau_{0}} \\
\frac{s}{h} & -s
\end{array}\right)\right] E_{1} \\
& =\tau_{0}\left(\begin{array}{cc}
2 i w_{0}-r+\frac{2 r u^{*}}{K}+\frac{2 a m u^{*} v^{*}}{\left(a+u^{* 2}\right)^{2}} & \frac{m u^{*}}{a+u^{* 2}} e^{-2 i w_{0} \tau_{0}} \\
-\frac{s}{h} & 2 i w_{0}+s
\end{array}\right) E_{1} \text {. }
\end{aligned}
$$

Therefore, it is possible to obtain

$$
E_{1}=E_{11} \cdot E_{12},
$$

where

$$
\begin{gathered}
E_{11}=\left(\begin{array}{cc}
2 i w_{0}-r+\frac{2 r u^{*}}{K}+\frac{2 a m u^{*} v^{*}}{\left(a+u^{* 2}\right)^{2}} & \frac{m u^{*}}{a+u^{* 2}} e^{-2 i w_{0} \tau_{0}} \\
-\frac{s}{h} & 2 i w_{0}+s
\end{array}\right)^{-1}, \\
E_{12}=2\left(\begin{array}{c}
-\frac{r}{K}-b_{20}-b_{11} C e^{-i w_{0} \tau_{0}} \\
-\frac{s}{h u^{*}}-\frac{s h}{u^{*}} C^{2}+\frac{2 s}{u^{*}} C
\end{array}\right) .
\end{gathered}
$$

Similarly,

$$
\begin{aligned}
H_{11}(0)= & -\left[g_{11} q(0)+\bar{g}_{11} \bar{q}(0)\right] \cdot f_{0}+\tau_{0} \\
& \times\left(\begin{array}{c}
2\left(-\frac{r}{K}-b_{20}\right)-b_{11}\left(C e^{-i w_{0} \tau_{0}}+\bar{C} e^{i w_{0} \tau_{0}}\right) \\
-\frac{2 s}{h u^{*}}-\frac{2 s h}{u^{*}} C \bar{C}+\frac{2 s}{u^{*}}(C+\bar{C})
\end{array}\right) .
\end{aligned}
$$




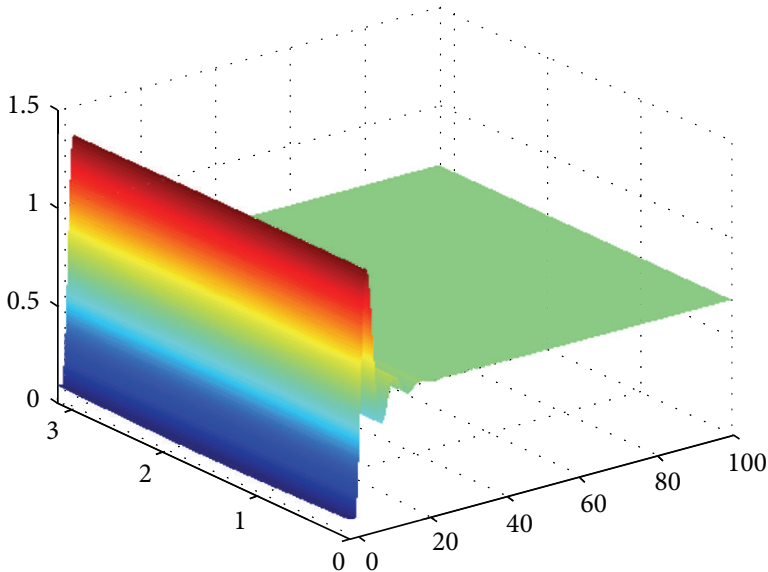

(a)

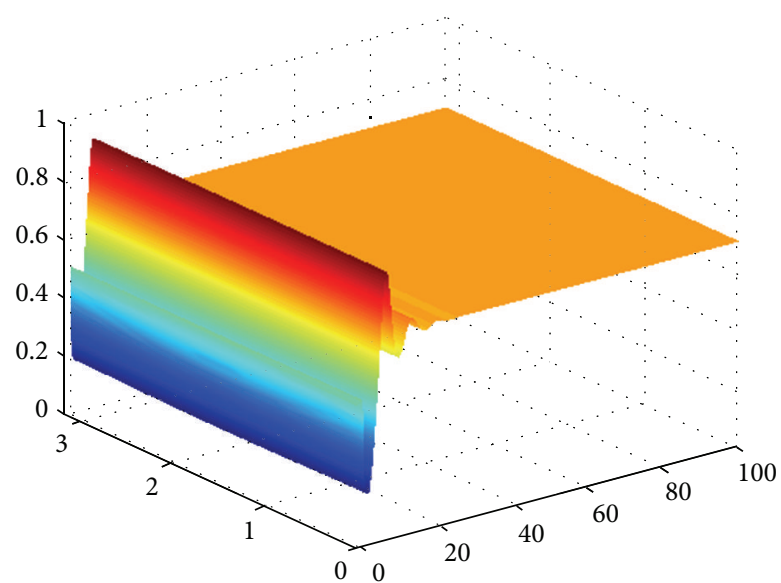

(b)

FIGURE 1: Convergence to positive equilibrium. Here the parameters are assumed to be $\tau_{1}=0, \tau_{2}=1.5$, and the initial values are $u(x, t)=$ $0.1+0.01 t \sin x, v(x, t)=0.5+0.01 t \cos x$, and $t \in[-1.5,0], x \in[0, \pi]$.

Then

$$
\begin{aligned}
\tau_{0}\left(\begin{array}{c}
2\left(-\frac{r}{K}-b_{20}\right)-b_{11}\left(C e^{-i w_{0} \tau_{0}}+\bar{C} e^{i w_{0} \tau_{0}}\right) \\
-\frac{2 s}{h u^{*}}-\frac{2 s h}{u^{*}} C \bar{C}+\frac{2 s}{u^{*}}(C+\bar{C})
\end{array}\right) \\
=g_{11} q(0)+\bar{g}_{11} \bar{q}(0)-\int_{-1}^{0} d \eta(\theta, 0) W_{11}(\theta) \\
=g_{11} q(0)+\bar{g}_{11} \bar{q}(0) \\
\quad-\int_{-1}^{0} d \eta(\theta, 0)\left[\frac{-i g_{11} q(\theta)}{w_{0} \tau_{0}}+\frac{i \bar{g}_{11} \bar{q}(\theta)}{w_{0} \tau_{0}}+E_{2}\right] \\
=-\int_{-1}^{0} d \eta(\theta, 0) E_{2} \\
=\tau_{0}\left(\begin{array}{cc}
-r+\frac{2 r u^{*}}{K}+\frac{2 a m u^{*} v^{*}}{\left(a+u^{* 2}\right)^{2}} & \frac{m u^{*}}{a+u^{* 2}} \\
-\frac{s}{h} & s
\end{array}\right) E_{2} .
\end{aligned}
$$

Therefore, it is possible to obtain

$$
E_{2}=E_{21} \cdot E_{22}
$$

where

$$
\begin{gathered}
E_{21}=\left(\begin{array}{cc}
-r+\frac{2 r u^{*}}{K}+\frac{2 a m u^{*} v^{*}}{\left(a+u^{* 2}\right)^{2}} & \frac{m u^{*}}{a+u^{* 2}} \\
-\frac{s}{h}
\end{array}\right)^{-1}, \\
E_{22}=\left(\begin{array}{c}
2\left(-\frac{r}{K}-b_{20}\right)-b_{11}\left(C e^{-i w_{0} \tau_{0}}+\bar{C} e^{i w_{0} \tau_{0}}\right) \\
-\frac{2 s}{h u^{*}}-\frac{2 s h}{u^{*}} C \bar{C}+\frac{2 s}{u^{*}}(C+\bar{C})
\end{array}\right) .
\end{gathered}
$$

Then $g_{21}$ is expressed by the parameters. Therefore, the following values can be computed:

$$
\begin{gathered}
c_{1}(0)=\frac{i}{2 w_{0} \tau_{0}}\left(g_{20} g_{11}-2\left|g_{11}\right|^{2}-\frac{\left|g_{02}\right|^{2}}{3}\right)+\frac{g_{21}}{2}, \\
\mu_{2}=-\frac{\operatorname{Re}\left(c_{1}(0)\right)}{\operatorname{Re} \lambda^{\prime}\left(\tau_{0}\right)}, \\
\beta_{2}=2 \operatorname{Re}\left(c_{1}(0)\right), \\
T_{2}=-\frac{\operatorname{Im}\left\{c_{1}(0)\right\}+\mu_{2} \operatorname{Im}\left\{\lambda^{\prime}\left(\tau_{0}\right)\right\}}{w_{0} \tau_{0}} .
\end{gathered}
$$

Theorem 6. If $\mu_{2}, \beta_{2}$, and $T_{2}$ are defined as above, then the following are true:

(i) if $\mu_{2}>0\left(\mu_{2}<0\right)$, then the Hopf bifurcation is supercritical (subcritical);

(ii) if $\beta_{2}<0\left(\beta_{2}>0\right)$, then the periodic solutions are stable (unstable);

(iii) if $T_{2}>0\left(T_{2}<0\right)$, then the period of the bifurcating periodic solutions of system (3) increases (decreases).

Remark 7. The direction and stability of the Hopf bifurcation have already been computed when $\tau=\tau_{0}$. The other Hopf bifurcation value $\tau_{k}$ can be analyzed using the same procedure.

\section{Simulations and Discussion}

This paper has considered a delayed predator-prey model with modified Holling-Tanner functional response. The effect of time delays on the positive equilibrium of system (3) has been investigated. The results obtained here show that the time delay plays an important role in determining system stability. Theorem 3 shows that the time delay can cause a stable equilibrium to become unstable or, in other words, that 


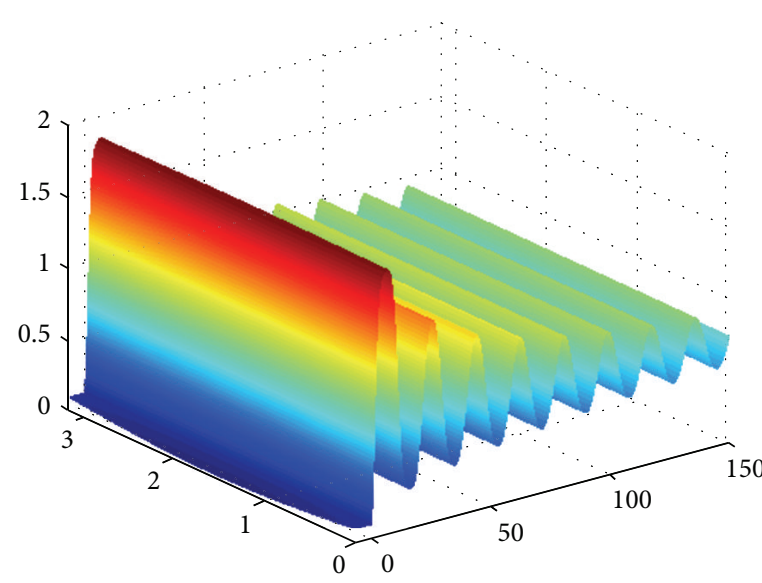

(a)

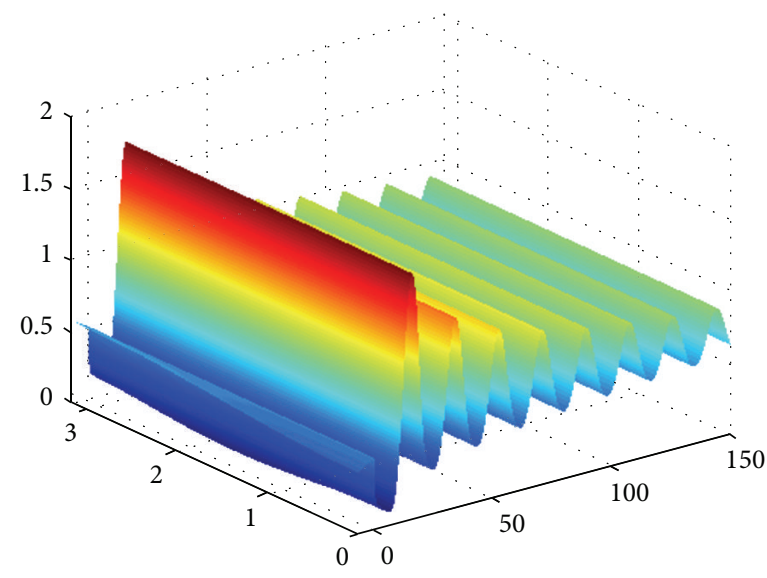

(b)

FIGURE 2: Convergence to positive equilibrium. Here the parameters are assumed to be $\tau_{1}=0, \tau_{2}=7$, and the initial values $u(x, t)=$ $0.1+0.01 t \sin x, v(x, t)=0.5+0.01 t \cos x$, and $t \in[-7,0], x \in[0, \pi]$.

there is a critical value $\tau_{0}$, such that, for $0 \leq \tau_{1}+\tau_{2}<\tau_{0}$, equilibrium $\left(u^{*}, v^{*}\right)$ is stable; when $\tau_{1}+\tau_{2}$ approaches $\tau_{0}$, equilibrium $\left(u^{*}, v^{*}\right)$ bifurcates into periodic solutions; and when $\tau_{1}+\tau_{2}>\tau_{0}$, the equilibrium becomes unstable.

Numerical simulations are performed to illustrate the dynamic behavior of system (3). Assume that $r=1, a=1$, $h=1, K=2, s=1, m=4, d_{1}=0.1, d_{2}=0.2$, and $l=1$. In this case, $\tau_{0}=2.201$. As shown in Figure 1, the positive equilibrium is stable when $\tau_{1}+\tau_{2}<\tau_{0}$, and the predators and prey tend to a steady state. However, if $\tau_{1}+\tau_{2}>\tau_{0}$, the positive equilibrium loses its stability and Hopf bifurcation occurs, leading to oscillatory behavior. These results are identical to the numerical results illustrated in Figure 2.

In both figures, the upper diagram represents the prey and the lower diagram represents the predator.

\section{Conflict of Interests}

The authors declare that there is no conflict of interests regarding the publication of this paper.

\section{Acknowledgments}

This work was supported by the Key Program of Zhejiang Provincial Natural Science Foundation of China (Grant no. LZ12C03001), by the National Natural Science Foundation of China (Grant no. 31370381), by the National Key Basic Research Program of China (973 Program, Grant no. 2012CB426510), and by the Zhejiang Provincial Natural Science Foundation of China (Grant no. LY13A010010).

\section{References}

[1] S. Yuan and Y. Song, "Stability and Hopf bifurcations in a delayed Leslie-Gower predator-prey system," Journal of Mathematical Analysis and Applications, vol. 355, no. 1, pp. 82-100, 2009.
[2] G. Hu and W. Li, "Hopf bifurcation analysis for a delayed predator-prey system with diffusion effects," Nonlinear Analysis: Real World Applications, vol. 11, no. 2, pp. 819-826, 2010.

[3] H. Shi, W. Li, and G. Lin, "Positive steady states of a diffusive predator-prey system with modified Holling-Tanner functional response," Nonlinear Analysis: Real World Applications, vol. 11, no. 5, pp. 3711-3721, 2010.

[4] S. Jana, M. Chakraborty, K. Chakraborty, and T. K. Kar, "Global stability and bifurcation of time delayed prey-predator system incorporating prey refuge," Mathematics and Computers in Simulation, vol. 85, pp. 57-77, 2012.

[5] Y. Zhu, Y. Cai, S. Yan, and W. Wang, "Dynamical analysis of a delayed reaction-diffusion predator-prey system," Abstract and Applied Analysis, vol. 2012, Article ID 323186, 23 pages, 2012.

[6] M. Zhao and S. Lv, "Chaos in a three-species food chain model with a Beddington-DeAngelis functional response," Chaos, Solitons and Fractals, vol. 40, no. 5, pp. 2305-2316, 2009.

[7] M. Zhao, H. Yu, and J. Zhu, "Effects of a population floor on the persistence of chaos in a mutual interference host-parasitoid model," Chaos, Solitons and Fractals, vol. 42, no. 2, pp. 12451250, 2009.

[8] S. Lv and M. Zhao, "The dynamic complexity of a three species food chain model," Chaos, Solitons and Fractals, vol. 37, no. 5, pp. 1469-1480, 2008.

[9] L. Zhu and M. Zhao, "Dynamic complexity of a host-parasitoid ecological model with the Hassell growth function for the host," Chaos, Solitons and Fractals, vol. 39, no. 3, pp. 1259-1269, 2009.

[10] L. Zhang and M. Zhao, "Dynamic complexities in a hyperparasitic system with prolonged diapause for host," Chaos, Solitons and Fractals, vol. 42, no. 2, pp. 1136-1142, 2009.

[11] H. Yu, M. Zhao, S. Lv, and L. Zhu, "Dynamic complexities in a parasitoid-host-parasitoid ecological model," Chaos, Solitons and Fractals, vol. 39, no. 1, pp. 39-48, 2009.

[12] C. Dai, M. Zhao, and L. Chen, "Complex dynamic behavior of three-species ecological model with impulse perturbations and seasonal disturbances," Mathematics and Computers in Simulation, vol. 84, pp. 83-97, 2012.

[13] D. J. Wollkind and J. A. Logan, “Temperature-dependent predator-prey mite ecosystem on apple tree foliage," Journal of Mathematical Biology, vol. 6, no. 3, pp. 265-283, 1978. 
[14] D. J. Wollkind, J. B. Collings, and J. A. Logan, "Metastability in a temperature-dependent model system for predator-prey mite outbreak interactions on fruit trees," Bulletin of Mathematical Biology, vol. 50, no. 4, pp. 379-409, 1988.

[15] R. M. May, Stability and Complexity in Model Ecosystems, Princeton University Press, Princeton, NJ, USA, 1978.

[16] Y. Li and C. Li, "Stability and Hopf bifurcation analysis on a delayed Leslie-Gower predator-prey system incorporating a prey refuge," Applied Mathematics and Computation, vol. 219, no. 9, pp. 4576-4589, 2013.

[17] C. Zhang, X. Yan, and G. Cui, "Hopf bifurcations in a predatorprey system with a discrete delay and a distributed delay," Nonlinear Analysis: Real World Applications, vol. 11, no. 5, pp. 4141-4153, 2010.

[18] F. Lian and Y. Xu, "Hopf bifurcation analysis of a predatorprey system with Holling type IV functional response and time delay," Applied Mathematics and Computation, vol. 215, no. 4, pp. 1484-1495, 2009.

[19] Y. Chen and F. Zhang, "Dynamics of a delayed predatorprey model with predator migration," Applied Mathematical Modelling, vol. 37, no. 3, pp. 1400-1412, 2013.

[20] W. Zuo and J. Wei, "Stability and Hopf bifurcation in a diffusive predatory-prey system with delay effect," Nonlinear Analysis: Real World Applications, vol. 12, no. 4, pp. 1998-2011, 2011.

[21] X. Lian, S. Yan, and H. Wang, "Pattern formation in predatorprey model with delay and cross diffusion," Abstract and Applied Analysis, Article ID 147232, 10 pages, 2013.

[22] M. Zhao, X. Wang, H. Yu, and J. Zhu, "Dynamics of an ecological model with impulsive control strategy and distributed time delay," Mathematics and Computers in Simulation, vol. 82, no. 8, pp. 1432-1444, 2012.

[23] H. Yu, M. Zhao, and R. P. Agarwal, "Stability and dynamics analysis of time delayed eutrophication ecological model based upon the Zeya reservoir," Mathematics and Computers in Simulation, vol. 97, pp. 53-67, 2014.

[24] F. Wei and Y. Cai, "Global asymptotic stability of stochastic nonautonomous Lotka-Volterra models with infinite delay," Abstract and Applied Analysis, vol. 2013, Article ID 351676, 8 pages, 2013.

[25] Y. Zhai, H. Bai, Y. Xiong, and X. Ma, "Hopf bifurcation analysis for the modified Rayleigh price model with time delay," Abstract and Applied Analysis, vol. 2013, Article ID 290497, 6 pages, 2013.

[26] R. Shi, J. Qi, and S. Tang, "Stability and bifurcation analysis for a predator-prey model with discrete and distributed delay," Abstract and Applied Analysis, vol. 2013, Article ID 454097, 12 pages, 2013.

[27] C. V. Pao, "Dynamics of nonlinear parabolic systems with time delays," Journal of Mathematical Analysis and Applications, vol. 198, no. 3, pp. 751-779, 1996.

[28] C. V. Pao, "Convergence of solutions of reaction-diffusion systems with time delays," Nonlinear Analysis: Theory, Methods \& Applications, vol. 48, no. 3, pp. 349-362, 2002.

[29] Q. X. Ye and Z. Y. Li, Introduction to Reaction-Diffusion Equations, Science Press, Beijing, China, 1990.

[30] B. D. Hassard, N. D. Kazarinoff, and Y. Wan, Theory and Applications of Hopf bifurcation, vol. 41 of London Mathematical Society Lecture Note Series, Cambridge University Press, Cambridge, UK, 1981.

[31] J. Wu, Theory and Applications of Partial Functional-Differential Equations, vol. 119 of Applied Mathematical Sciences, Springer, New York, NY, USA, 1996.
[32] X. D. Lin, J. W. So, and J. H. Wu, "Centre manifolds for partial differential equations with delays," Proceedings of the Royal Society of Edinburgh A: Mathematics, vol. 122, no. 3-4, pp. 237254, 1992.

[33] T. Faria, "Normal forms and Hopf bifurcation for partial differential equations with delays," Transactions of the American Mathematical Society, vol. 352, no. 5, pp. 2217-2238, 2000.

[34] T. Faria and L. T. Magalhães, "Normal forms for retarded functional-differential equations with parameters and applications to Hopf bifurcation," Journal of Differential Equations, vol. 122, no. 2, pp. 181-200, 1995.

[35] T. Faria and L. T. Magalhaes, "Normal forms for retarded functional-differential equations and applications to BogdanovTakens singularity," Journal of Differential Equations, vol. 122, no. 2, pp. 201-224, 1995. 


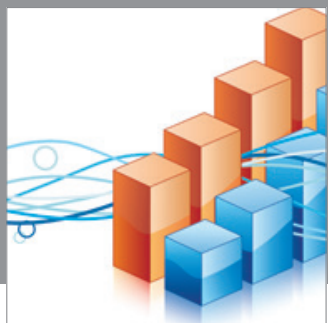

Advances in

Operations Research

mansans

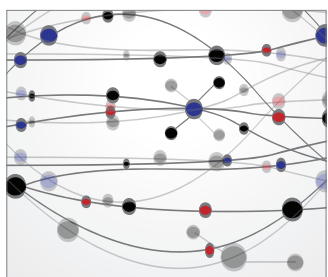

The Scientific World Journal
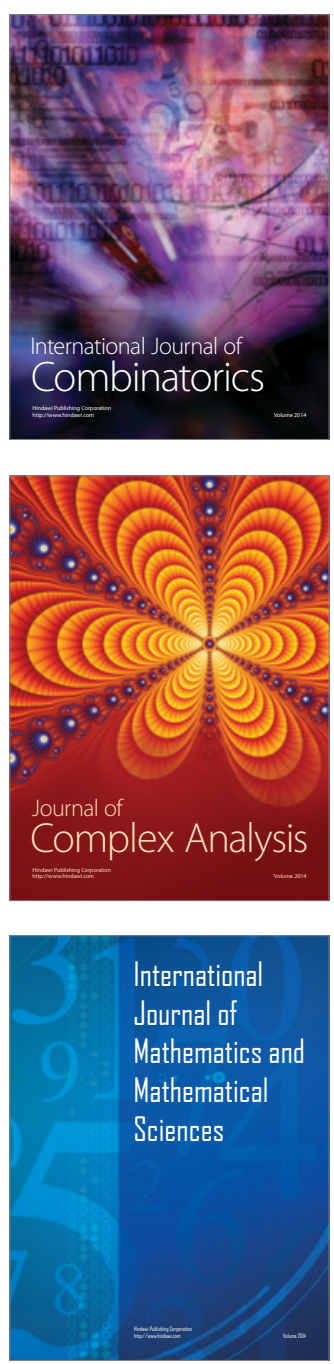
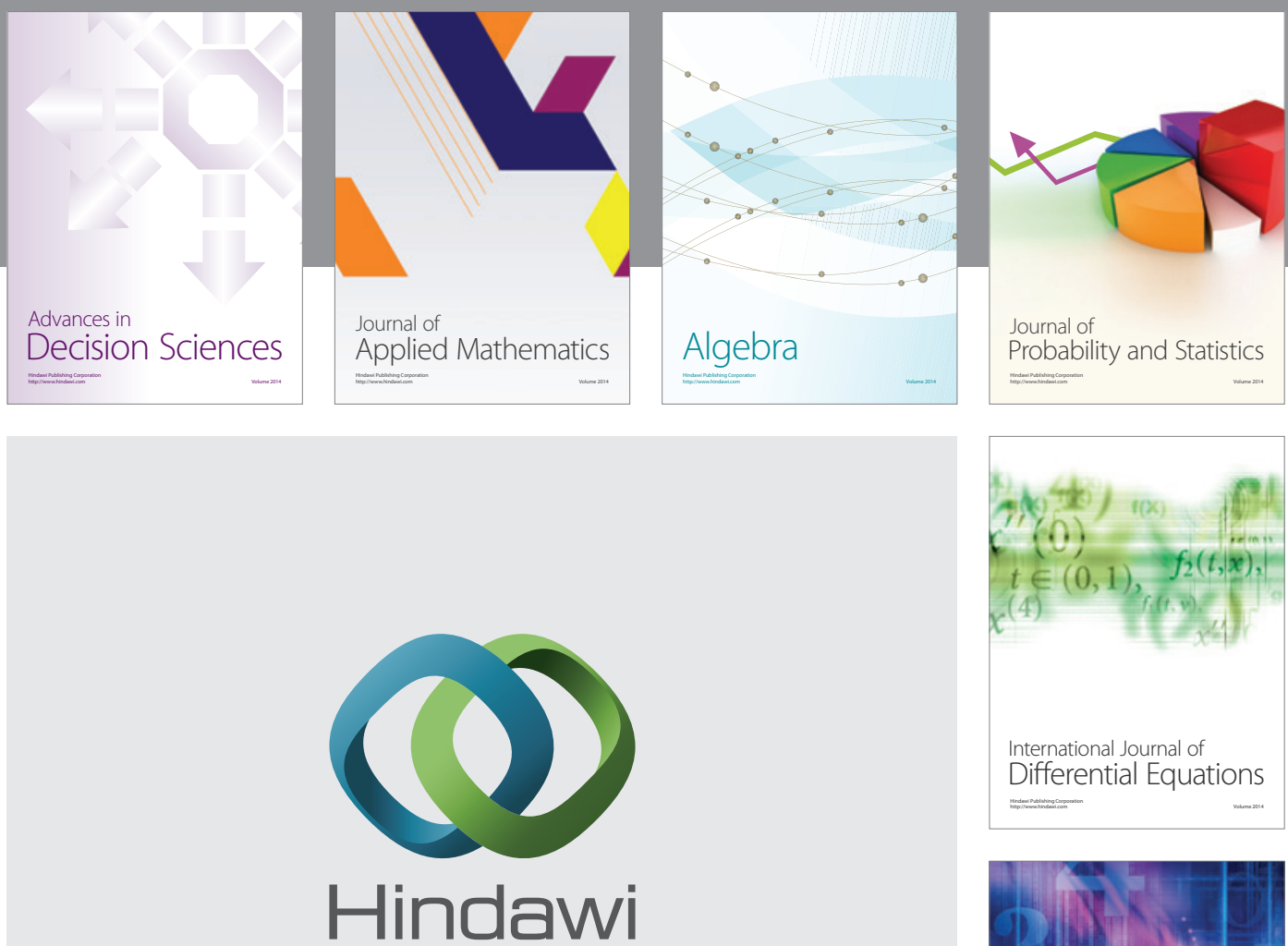

Submit your manuscripts at http://www.hindawi.com
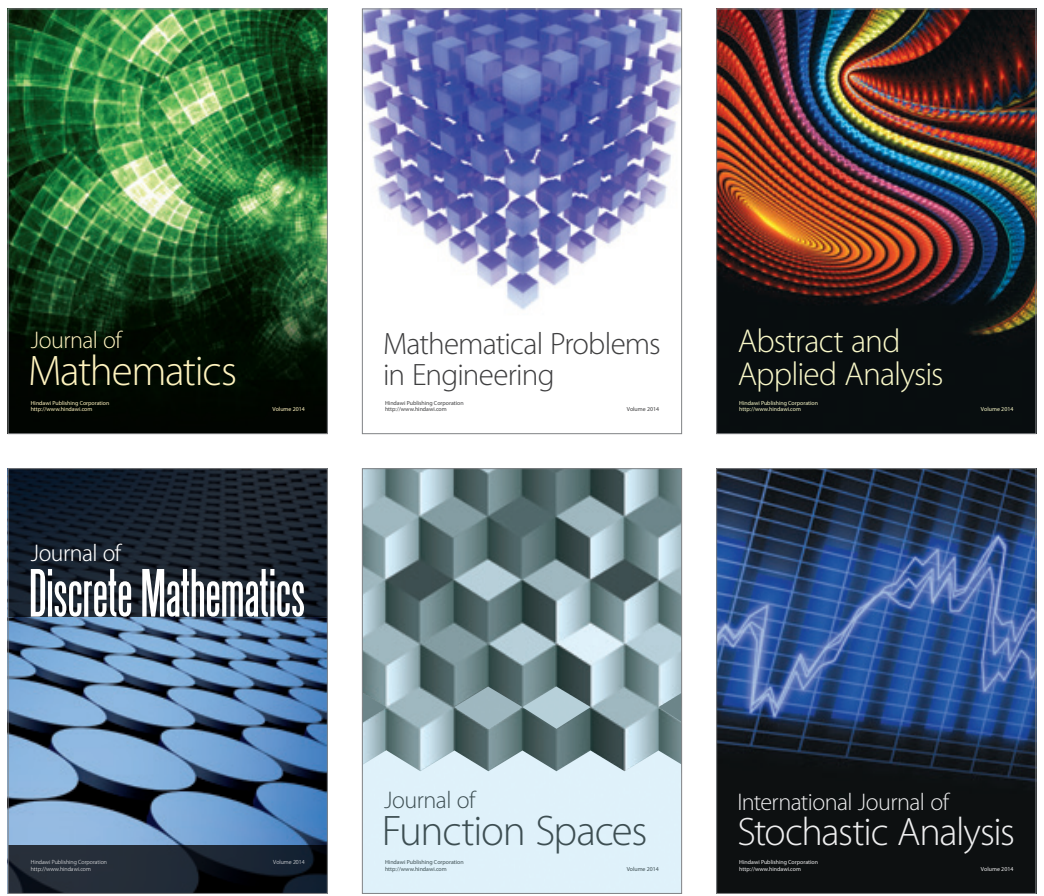

Journal of

Function Spaces

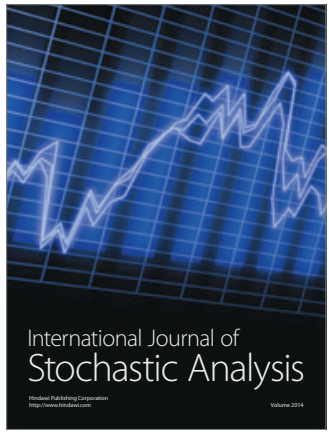

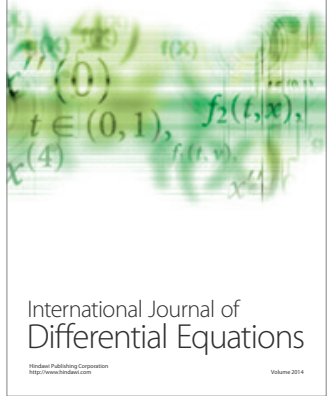
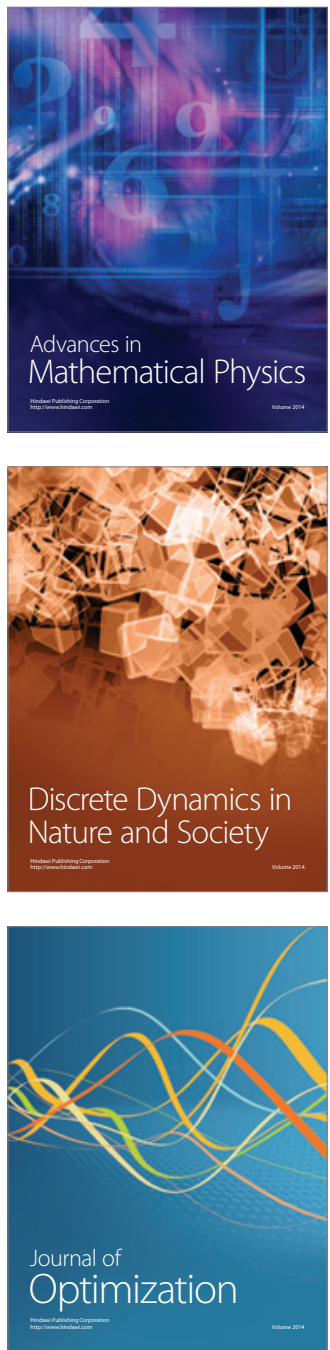